\title{
Article
}

\section{Frequency of post-stroke pneumonia: Systematic review and meta-analysis of observational studies}

Badve, M, Zhou, Z, van de Beek, D, Anderson, C and Hackett, Maree

Available at http://clok.uclan.ac.uk/24739/

Badve, M, Zhou, Z, van de Beek, D, Anderson, $C$ and Hackett, Maree ORCID: 0000-0003-1211-9087 (2019) Frequency of post-stroke pneumonia:

Systematic review and meta-analysis of observational studies. International Journal of Stroke, 14 (2). pp. 125-136. ISSN 1747-4930

It is advisable to refer to the publisher's version if you intend to cite from the work. $10.1177 \% 2 F 1747493018806196$

For more information about UCLan's research in this area go to http://www.uclan.ac.uk/researchgroups/ and search for <name of research Group>.

For information about Research generally at UCLan please go to http://www.uclan.ac.uk/research/

All outputs in CLoK are protected by Intellectual Property Rights law, including Copyright law. Copyright, IPR and Moral Rights for the works on this site are retained by the individual authors and/or other copyright owners. Terms and conditions for use of this material are defined in the policies page.

\section{CLoK}

Central Lancashire online Knowledge www.clok.uclan.ac.uk

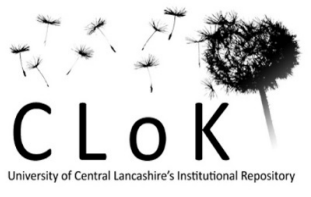


Frequency of post-stroke pneumonia: systematic review and meta-analysis of observational studies

Brief title: Frequency of post-stroke pneumonia

Word count: Abstract: 250 words; Title page, abstract, full-text, references, legends, figures, tables: $5 \underline{397397}$ words (max 5000 words)

\section{Authors}

Monica S. Badve, D.N.B. ${ }^{1,2,3}$, Zien Zhou, M.D. ${ }^{2,4}$, Diederik van de Beek Ph.D. ${ }^{5}$,

Craig S. Anderson, Ph.D. ${ }^{3,6,7,8}$, Maree L. Hackett, Ph.D. 2,3,9

\section{Author affiliations}

1. Department of Neurology, The St George hospital, Kogarah, Australia

2. Mental Health Program, The George Institute for Global Health, Faculty of Medicine, University of New South Wales, Sydney, Australia

3. School of Medicine, The University of Sydney, Camperdown, Sydney, Australia

4. Department of Radiology, Ren Ji Hospital, School of Medicine, Shanghai Jiao Tong University, Shanghai, China

5. University of Amsterdam, Amsterdam University Medical Centers, Amsterdam Neuroscience, Meibergdreef, Amsterdam, The Netherlands

6. Cardiometabolic cluster, The George Institute for Global Health, Faculty of Medicine, UNSW, Sydney, Australia

7. The George Institute China at Peking University Health Science Center, Beijing, China

8. Neurology Department, Royal Prince Alfred Hospital, Camperdown, Australia

9. Faculty of Health and Wellbeing, The University of Central Lancashire, Preston, United Kingdom 
Email : monica.badve@health.nsw.gov.au 


\section{ABSTRACT}

Background: Post-stroke pneumonia and other infectious complications are serious conditions whose frequency varies widely across studies.

Aims: We conducted a systematic review to estimate the frequency of post-stroke pneumonia and other types of major infection.

Summary of review: MEDLINE, EMBASE, CINAHL and PsycINFO databases were searched for prospective studies with consecutive recruitment of stroke patients. The primary outcome was post-stroke pneumonia. Secondary outcomes were any infection and urinary tract infection. Quality assessment was done using Newcastle Ottawa scale. Heterogeneity of estimates across study populations was calculated using Cochran's Q (heterogeneity $\chi^{2}$ ) and $\mathrm{I}^{2}$ statistics. A total of 47 studies (139,432 patients) with 48 sample populations were eligible for inclusion. Mean age of patients was 68.3 years and their mean National Institute of Health Stroke Scale score was 8.2. The pooled frequency of post-stroke pneumonia was $12.3 \%\left(95 \%\right.$ confidence interval $\left.[\mathrm{CI}] 11 \%-13.6 \% ; \mathrm{I}^{2}=98 \%\right)$. The pooled frequency from 2011 to 2017 was $13.5 \%$ (95\% CI $11.8 \%-15.3 \%$; $\mathrm{I}^{2}=98 \%$ ) and comparable with earlier periods $(\mathrm{P}$ interaction $=0.31)$. The pooled frequency in studies in stroke units was $8 \%(95 \%$ CI 7.1\%-9\%; $\mathrm{I}^{2}=78 \%$ ) and significantly lower than other locations (P interaction=0.001). The pooled frequency of post-stroke infection was $21 \%\left(95 \% \mathrm{CI} 13 \%-29.3 \% ; \mathrm{I}^{2}=99 \%\right)$ and of post-stroke urinary tract infection was $7.9 \%\left(95 \%\right.$ CI $\left.6.7 \%-9.3 \% ; \mathrm{I}^{2}=96 \%\right)$.

Conclusion: Approximately 1 in 10 stroke patients experience pneumonia during the acute period of hospital care. The frequency of post-stroke pneumonia has remained stable in recent decades but is lower in patients receiving stroke unit care compared to management in other ward settings. 


\section{INTRODUCTION}

Stroke is the second leading cause of death in high-income countries ${ }^{1-3}$. The overall burden due to stroke is even greater in middle and low-income countries ${ }^{3,4}$. The global burden of disease study suggests that stroke will remain one of the leading causes of disability-adjusted life years (DALYs) in the near future. Pneumonia is the major early complication of stroke and is associated with high morbidity and mortality ${ }^{5,6}$. Pneumonia by itself is also associated with poor functional outcomes and the prevalence has varied from 5 to 10 patients per 1000 population ${ }^{7}$. The frequency of post-stroke pneumonia varies widely within and over time in the literature with ranges reported from $1.4 \%$ to $57 \%{ }^{8,9}$.

While major risk factors for post-stroke pneumonia include older age, dysphagia, male gender, stroke severity, pre-admission dependency, coronary artery disease, congestive cardiac failure, and chronic obstructive pulmonary disease, it has also been regarded as a marker of stroke severity ${ }^{10,11}$. In the last 10 years, there have been enormous advances in primary and secondary stroke and care, including an emphasis on stroke unit care ${ }^{12,13}$. We undertook a systematic review of the frequency of post-stroke pneumonia and its relationship with pathological stroke subtype and type of stroke care over time.

\section{METHODS}

This systematic review was conducted according to the MOOSE guidelines and reported according to the Preferred Reporting Items for Systematic Reviews and Meta-Analyses (PRISMA) ${ }^{14-16}$. The protocol is registered in the International prospective register of systematic reviews (PROSPERO) (CRD20171221) at https://www.crd.york.ac.uk/PROSPEROFILES/78368_PROTOCOL_20171221.pdf (see supplementary files) 
Search strategy and selection criteria

MEDLINE (Medical Literature Analysis and Retrieval System Online) via Ovid, EMBASE (Excerpta Medical Database) and CENTRAL (Cochrane Central Register of Controlled Trials), CINAHL (Cumulative index to nursing and allied health literature) and PsycINFO were searched from inception to October 2017 using search strategies specified in the registered protocol. Studies were eligible for inclusion if they: (1) were prospective cohort studies with consecutive recruitment; (2) involved adult patients (age $\geq 18$ years) admitted within 30 days of acute ischaemic or haemorrhagic stroke; and (3) reported the proportion of patients with post-stroke pneumonia. There were no language restrictions or study size exclusions. Studies were excluded if they were randomised controlled trials, casecontrol or cross-sectional studies or had retrospective recruitment. Studies with overlapping recruitment sites, study dates, funding grant numbers and similar patient characteristics were considered to be from the same cohort and in that case the study with the largest cohort was included. This occurred in three instances 5,9,17.

Data extraction and quality assessment

Two authors (M.S.B. and Z.Z.) independently evaluated all citations by title and abstract and retrieved the full text in detail for those that appeared relevant. One author (M.S.B) extracted data which were checked by another author (Z.Z). Methodological quality of the included studies was assessed using the Newcastle Ottawa scale including assessment of the representativeness of the cohort ${ }^{18}$. Since this was a study of frequency of post-stroke pneumonia, exposure was not studied and selection of the unexposed cohort, ascertainment of exposure and comparability of cohorts on the basis of design or analysis were not applicable. Demonstration that post-stroke pneumonia was not present at the outset, assessment of outcome and follow-up length and adequacy of cohort follow-up were assessed. Since poststroke pneumonia usually is an acute medical complication not requiring prolonged 
observation or follow-up, all studies with in-hospital observation of post-stroke pneumonia were defined as having an adequate (one star) follow-up length. Studies with follow-up postdischarge were considered to have a good follow up.

\section{Outcome measures}

The primary study outcome was the frequency of post-stroke pneumonia defined by any diagnostic method. Secondary outcomes were frequencies of post-stroke infections, and urinary tract infection after acute stroke. The authors' criteria for the diagnosis of post-stroke pneumonia were based on history and clinical features (cough, purulent secretions, fever, leucocytosis), infiltrates on chest x-ray, staining and culture of the causative microorganism, and frequently were also based on established criteria for the diagnosis of post-stroke pneumonia, Mann criteria and Centre for Disease control (CDC) criteria (Online supplementary files). Ventilator-associated pneumonia was defined as pneumonia developing in patients receiving mechanical ventilation. The authors' criteria for the diagnosis of pneumonia, infection and urinary tract infection were accepted.

Dysphagia assessment was done by doctors and/or nurses and/or specialist speech pathologists (speech and language therapists). Criteria used in studies included were clinical, and/or radiologic and/or endoscopic. History and clinical examination including assessment of bulbar function, testing for dysarthria, dysphonia and voluntary cough, symmetry and speed of tongue and palatal movements were done to diagnose dysphagia clinically. Clinical criteria used to diagnose dysphagia were the swallowing provocation test, water swallow test, staged water swallow test, three-step swallowing screen protocol, Paramatta Hospital assessment of dysphagia and ROSS test (Online supplementary files, Supplementary Table 1). The radiologic methods used were the modified barium swallow or videofluoroscopic evaluation of swallowing (VFES). The endoscopic methods used to diagnose dysphagia were fibreoptic endoscopic evaluation of swallowing (FEES). 
Subgroup analyses were conducted according to location (stroke unit and ward [including patients treated in a combination of stroke unit and ward or neurology ward or medical ward], only stroke unit, intensive care unit [ICU] with ward and emergency department), according to type of stroke (only ischaemic stroke, ischaemic stroke and intracerebral haemorrhage and only intracerebral haemorrhage), according to the aim of the study (studies specifically evaluating infection after acute stroke versus other studies), according to era (before 1990, 1991-2000, 2001-2010 and 2011onward ) and according to interventions received by the patient populations (intravenous thrombolysis, intra-arterial interventions, intensive care unit interventions, nasogastric tubes and all other interventions). We extracted data for dependence and stroke severity if the National Institute of Health stroke scale (NIHSS) was used (see supplementary files).

Data Synthesis and Analysis

Pooled proportion of post-stroke pneumonia was calculated using the random-effects approach of DerSimonian and Laird ${ }^{19}$. Sub-group meta-analyses of proportion with poststroke pneumonia were conducted as described earlier. Heterogeneity of the frequency of post-stroke pneumonia between study populations was calculated using Cochran's Q (heterogeneity $\chi^{2}$ ) and $\mathrm{I}^{2}$ statistics ${ }^{20} . \mathrm{I}^{2}$ values of less than $25 \%, 50 \%$ to less than $75 \%$ and more than $75 \%$ were regarded as evidence of low, moderate and high levels of inconsistency respectively ${ }^{20}$. Subgroups were compared using random-effects meta-regression analyses to assess the effects of type of stroke, location of patient, era, World Bank country/region economy ranking, type of study and intervention used on the frequency of post-stroke pneumonia. Publication bias was assessed by Begg and Mazumdar rank correlation test ${ }^{21}$. Analyses were conducted using Stata/MP (version 14.2, Stat Corp, College Station, Texas). 
Role of funding source

This study had no funding. The corresponding author had access to all data and took final responsibility for submission of this paper.

\section{RESULTS}

Selection and description of studies

Electronic searching retrieved 2331 citations from MEDLINE, EMBASE, CINAHL and PsycINFO (Figure 1). Sixty studies were identified from reference lists and added to the original list. Three hundred and sixty duplicate citations were excluded leaving 2031 citations. After screening, 1826 were excluded, and 205 full text articles were assessed for eligibility. A total of 48 sample populations in 47 studies were eligible and included in the meta-analyses. Forty-seven studies including 139,432 stroke patients met the inclusion criteria (Table 1; Supplementary Table 2).

Median study size was 310 (range 100- 63650) and mean study size was 2966 (Table 1). Studies were primarily conducted in the ward and stroke unit setting (28 studies;111409 participants), followed by only in the stroke unit setting (14 studies; 26942 participants), ICU and ward setting (4 studies; 798 participants) and emergency department (1 study; 283 participants) (1). In the study populations, where data were reported, the mean age of participants was 68.3 years, $51.7 \%$ were male, and their mean baseline National Institute of Health Stroke Scale (NIHSS) score was 8.2. One fifth (22.7\%) of patients had dysphagia, $17 \%$ had nasogastric tubes (one study of 100 participants only included patients with nasogastric tubes ${ }^{22}$ ) and $17.3 \%$ had lowered consciousness ${ }^{22}$. The data on patients with nasogastric tubes and lowered consciousness were from separate studies except for one study ${ }^{22}$. The proportion of patients with dysphagia without lowered consciousness where data was 
available (5 studies) was $52.5 \%$ (Table 1 ). The number of days of observation in the hospital ranged from 2 to 21 days. Follow-up after discharge from hospital occurred in ten studies and this ranged from 14 to 90 days.

Quality assessment

All studies scored one star for representativeness of the cohort and assessment of outcome (Figure 2). Only 7 studies explicitly stated that pneumonia was not present at the start of the study. All studies scored one star for assessment of outcome. All studies scored one star indicating an adequate length of follow-up. However, in only ten studies did followup continue after discharge indicative of good follow-up length. There was adequate cohort follow-up only in the ten studies with follow-up continuing after discharge.

Pooled frequency of post-stroke pneumonia and subgroup analyses

The pooled frequency of post-stroke pneumonia in the included studies was $12.3 \%$ (95\% confidence interval $[\mathrm{CI}] 11.1 \%-13.6 \% ; \mathrm{I}^{2}=98 \%$ ) (Figure 3 ). The pooled frequency in the studies including only patients treated in stroke units (14 studies; 26,942 participants) was $8 \%\left(95 \%\right.$ CI $\left.7.1 \%-9 \% ; I^{2}=78 \%\right)$ and this was statistically less than the pooled frequency in patients treated in the ward and stroke unit (28 studies; 111,409 participants, $13.1 \%$ [95\% CI $\left.\left.11.3 \%-14.9 \% ; \mathrm{I}^{2}=98 \%\right]\right)(\mathrm{P} \text { interaction }=0.001)^{5,23-26}$. The pooled proportion of patients with post-stroke pneumonia in ICUs (4 studies; 798 participants) was 30.3\% (95\% CI 12.9\%$47.6 \% ; \mathrm{I}^{2}=97 \%$ ), and much higher than other pooled estimates based on patient location (Table 2) ${ }^{8,27-30}$. In ICU studies, the frequency of ventilator-associated post-stroke pneumonia in intubated and ventilated patients ( 2 studies; 36 participants) was 80.6\% (95\% CI 67.8\%$93.6 \% ; \mathrm{I}^{2}=0 \%$ ) and this was even higher than the frequency of post-stroke pneumonia in ICU patients. 
The pooled frequency of post-stroke pneumonia in patients with intracerebral haemorrhage (2 studies; 5423 participants) was $16.8 \%$ (95\% CI 15.8\%-17.8\%; $\left.\mathrm{I}^{2}=0 \%\right)$ and comparable with the pooled frequency of post-stroke pneumonia in patients with only ischaemic stroke (24 studies; 35252 participants, $11.7 \%$ [95\% CI 9.2\%-14.1\%; $\left.\mathrm{I}^{2}=98 \%\right]$ ) and patients with intracerebral haemorrhage and ischaemic stroke (22 studies; 98757 participants, $11.7 \%$ [95\% CI 10.3\%-13\%; $\left.\left.\mathrm{I}^{2}=96 \%\right]\right)(\mathrm{P}$ interaction $=0.37)(\text { Table } 2)^{31}$.

The pooled frequency of post-stroke pneumonia from 2011-2017 (17 studies; 106406 participants, $13.6 \%$ [95\% CI 11.8\%-15.3\%; $\mathrm{I}^{2}=98 \%$ ]) was comparable with 1991-2000 (5 studies; 734 participants, 9.9\% (95\% CI 5.46\%-14.29\%; $\mathrm{I}^{2}=78 \%$ ) and 2001-2010 (25 studies; 32188 participants, $11.8 \%$ (95\% CI 9.9\%-13.7\%; $\left.\mathrm{I}^{2}=97 \%\right)(\mathrm{P}$ interaction=0.31) (Table 2). There was only one study assessing the frequency of post-stroke pneumonia in a low-income country (148 participants, $18.2 \%$ [95\% CI 12\%-14.5\%]). This was comparable with the pooled frequencies in high-income countries or regions (38 studies; 114450 participants, $11.7 \%$ [95\% CI 10.4\%-13\%; $\left.\mathrm{I}^{2}=98 \%\right]$ ) and middle-income countries or regions (8 studies; 24834 participants, $13.8 \%$ [95\% CI 11.3\%-16.2\%; $\left.\left.\mathrm{I}^{2}=87 \%\right]\right)(\mathrm{P}$ interaction=0.77) (Table 2).

In studies aimed at specifically evaluating post-stroke infections (20 studies; 74187 participants) the pooled frequency was $16.5 \%\left(95 \% \mathrm{CI} 13.8 \%-19.2 \% ; \mathrm{I}^{2}=98 \%\right)$ which was higher than that found in other studies not aimed at specifically studying post-stroke infections (28 studies; 65245 participants, 10\% [95\% CI 8.7\%-11.4\%; $\left.\mathrm{I}^{2}=96 \%\right]$ ) and statistically significant $(\mathrm{P}$ interaction $=0.01)$ (Table 2$)$. The frequency of post-stroke pneumonia in patients with nasogastric tubes (1 study; 100 participants) was 44\% (95\% CI $34.2 \%-53.7 \%$ ) and the pooled frequency in patients receiving interventions in ICU (4 studies; 798 participants) was $30.2 \%$ (95\% CI $12.9 \%-47.6 \%$; $\mathrm{I}^{2}=97 \%$ ) and these were higher than $10.5 \%$ (95\% CI 9.2\%-11.7\%; $\mathrm{I}^{2}=98 \%$ ) in patients receiving other interventions (40 studies; 137831participants), intravenous thrombolysis (1 study; 538 participants, 22.7\% [95\% CI 
19.5\%-26.2\%]) and intra-arterial interventions ( 1 study; 165 participants, $13.3 \%$ [95\% CI

$8.1 \%-18.5 \%])$ and this was not a statistically significant interaction (P interaction $=0.5)($ Table

2). In the primary and sub-group analyses of the frequency of post-stroke pneumonia, heterogeneity was high $\left(\mathrm{I}^{2}=78 \%-98 \%\right)$ except for the 2 sample populations with intracerebral haemorrhage and ventilator-associated post-stroke pneumonia $\left(\mathrm{I}^{2}=0 \%\right)$.

Pooled frequency of post-stroke infections and post-stroke urinary tract infection

The pooled frequency of post-stroke infection (11 studies; 8581 participants) was $21 \%\left(95 \%\right.$ CI 13\%-29.3\%) (Figure 4) with high statistical heterogeneity $\left(\mathrm{I}^{2}=99 \%\right)$. The pooled frequency of post-stroke urinary tract infection (28 studies; 39282 participants) was 7.9\% (95\% CI 6.7\%-9.3\%) (Figure 5) with high statistical heterogeneity $\left(\mathrm{I}^{2}=96 \%\right)$.

\section{Meta-regression analysis and small study effects}

Significant interaction between the sub-groups of care location of patients (stroke unit and ward, stroke unit, ICU and ward, emergency), type of study (aim of study focussing on post-stroke infection and/or post-stroke pneumonia, other studies) were identified in metaregression. There was no statistically significant interaction for the other sub-group (type of stroke, era, country income and interventions used [ICU interventions, nasogastric tubes, intravenous thrombolysis, intra-arterial thrombolysis and/or clot retrieval, other interventions]) analyses. When funnel plots were examined for small study effects, these were symmetrical suggesting that there was no evidence of publication bias.

\section{DISCUSSION}

In this large systematic review and meta-analysis, the point estimate of the pooled frequency of post-stroke pneumonia was $12.3 \%$. The point estimate of pooled frequency of 
post-stroke pneumonia in the present era (2011 onward) remains unchanged from previous years. It is surprising that there is no evidence of a reduction in the frequency of post-stroke pneumonia over time given the great advances in stroke care and acute stroke therapies. This could possibly be explained by increased survival of patients with more severe strokes with improved stroke care ${ }^{32}$. Increased stroke severity leads to an increased tendency to develop post-stroke pneumonia due to dysphagia and depressed consciousness. The lack of reduction in the post-stroke pneumonia frequency could also be attributed to better awareness, methods of detection and reporting bias. The relatively small number of included patients receiving thrombolysis and/or clot retrieval may also explain the consistent frequency of post-stroke pneumonia over time. Over the last twenty-five years the absolute number of stroke survivors and people with new stroke has increased one to two- fold. Most of this increased burden is in developing countries yet most of the studies included in our review are from high-income countries ${ }^{33}$. However, the increase in stroke burden does not necessarily indicate that affected persons have increased stroke severity.

The pooled frequency of post-stroke pneumonia was higher in ICU patients and in the study exclusively including patents with nasogastric tubes, reflecting the inclusion of patients with more severe strokes, on ventilators and with an increased tendency to have medical complications including pneumonia. Among patients in ICUs with endotracheal intubation and mechanical ventilation, the frequency of ventilator- associated post-stroke pneumonia was much higher than other patients. Bundles are methods to implement best practices. The ventilator-associated pneumonia 'prevention' bundle can reduce the incidence of pneumonia by at least $50 \%{ }^{34}$. The prevention bundle mainly includes elevation of head of the bed by $30^{\circ}$ to $45^{\circ}$ (head elevation is not recommended if cerebral perfusion pressure is threatened), daily sedation interruption and assessment of readiness to extubate, use of subglottic secretion drainage and avoidance of scheduled ventilator circuit changes ${ }^{35-37}$. Other additions to this 
bundle are the use of oral chlorhexidine antiseptic to prevent pneumonia, peptic ulcer disease prophylaxis and deep vein thrombosis prophylaxis ${ }^{36}$. In the study exclusively including patients with nasogastric tubes the frequency of pneumonia was high probably since all the patients had dysphagia, one-fifth of these had lowered level of consciousness, and severe upper motor neuron facial palsy with paresis of the tongue and oropharyngeal muscles was seen in many patients ${ }^{22}$.

The pooled frequency of post-stroke pneumonia was comparable in populations of patients with intracerebral haemorrhage and patients with ischaemic stroke. This may be due to improvements in post-stroke care that despite the increased severity of the clinical presentation in patients with intracerebral haemorrhage, the frequency of post-stroke pneumonia is not significantly higher than patients with ischaemic stroke. The pooled frequency of post-stroke pneumonia was lowest in stroke units indicating that stroke unit care may be effective in preventing post-stroke pneumonia. This is probably due to better facilities and clinical protocols in place in stroke units including dysphagia management to prevent aspiration. The pooled frequency of post-stroke infection was higher than post-stroke pneumonia and apart from post-stroke pneumonia, this also included post-stroke urinary tract infections, acute bronchitis, upper respiratory tract infections, viral infections, fever of unknown origin and other infections ${ }^{38-45}$. The frequency of post-stroke urinary tract infections was similar to previous studies ${ }^{6}$.

This is the largest systematic review and meta-analysis including high quality studies with prospective, consecutive recruitment providing data on the frequency of post-stroke pneumonia. Scores on the Newcastle- Ottawa scale indicated fair to good quality for most studies with poor quality in very few instances in the domains studied. There are limited new data on the frequency of post-stroke infection, post-stroke pneumonia and urinary tract infection and research in this area is needed considering the poor outcomes associated ${ }^{6,46}$. 
We should acknowledge the clinical heterogeneity which included varying stroke populations with differing quality of stroke care and stroke severity. There are only two studies with patients with intracerebral haemorrhage indicative of overall bias in the pooled data for the frequency of post-stroke pneumonia, post-stroke infections and urinary tract infection after ischaemic stroke ${ }^{17,31}$. There are also limited data from middle-income and low-income countries/regions to inform clinical practice, probably due to the expense and infrastructure required to conduct prospective consecutive cohort studies over a reasonable time period. Since our search terms were primarily linked to respiratory infections, we may have missed studies with only data on the frequency of post-stroke infection and urinary tract infection.

There is scope for a large prospective cohort study of post-stroke pneumonia and post-stroke infections, in stroke units, in patients who have received thrombolysis and/or clot retrieval to determine whether the frequency of post-stroke pneumonia is lower in people receiving these interventions. Considering the expense of such a study, it may not be possible in a developing country, at least not over a long period of time.

In the last ten years, stroke has been named a prioritized noncommunicable disease by the World Health Organisation ${ }^{33}$. Given the unchanged frequency of post-stroke pneumonia in the present decade and the economic burden that it poses, this review indicates that there needs to be more robust practices in place for the prevention of post-stroke pneumonia globally. Though post-stroke pneumonia is an infection of the lung parenchyma due to invasion and overgrowth of micro-organisms, administration of prophylactic antibiotics has not been shown to improve outcomes of acute stroke patients in two recent large randomised trials ${ }^{47,48}$. Hence, after hospital admission of patients with acute stroke other appropriate preventive strategies including dysphagia screening, good nursing care, handwashing and aseptic precautions would need to be used to prevent pneumonia and other infections. In this systematic review, since stroke unit care was associated with the lowest frequency of post- 
stroke pneumonia, we would advocate for all patients with acute stroke to receive stroke unit care. Ventilator-associated pneumonia prevention bundles can help in reducing the incidence of pneumonia in ICU and we would recommend consideration of these bundles when patients with acute stroke are treated in ICUs. Primary and secondary stroke prevention may also play an important role in preventing post-stroke pneumonia by reducing stroke severity. Research on the predictors of post-stroke pneumonia and other infections would be useful to put appropriate preventive strategies in place.

Contributors: Conception and design: Monica Badve, Maree Hackett, Craig Anderson. Literature search and data extraction: Monica Badve, Zien Zhou. Analysis and interpretation of data: Monica Badve, Maree Hackett, Zien Zhou. Initial drafting of manuscript: Monica Badve. Critical revision of the manuscript for intellectual content: Monica Badve, Maree Hackett, Zien Zhou, Craig Anderson, Diederik van de Beek. Final approval of the manuscript: Monica Badve, Zien Zhou, Diederik van de Beek, Craig Anderson, Maree Hackett

Competing interests: We declare no competing interests.

Acknowledgements: The authors would like to thank Professor Suetonia Palmer for her help with this publication. During the completion of this review Zien Zhou held a Scientia PhD Scholarship from UNSW Sydney, Maree Hackett held a National Heart Foundation Future Leader Fellowship (2014-2017, 100034), and a NHMRC CDF fellowship APP1141328, 2018-2021, and Craig Anderson held a National Health and Medical Research Council (NHMRC) Senior Principal Research Fellowship. These sources of funding had no role in this study.

Funding: None 


\section{REFERENCES}

1. Feigin VL, Norrving B and Mensah GA. Global Burden of Stroke. Circ Res. 2017; 120: 439-48.

2. Thrift AG, Howard G, Cadilhac DA, et al. Global stroke statistics: An update of mortality data from countries using a broad code of "cerebrovascular diseases". Int J Stroke. 2017; 12: 796-801.

3. Collaborators GBDM. Global, regional, and national under-5 mortality, adult mortality, age-specific mortality, and life expectancy, 1970-2016: a systematic analysis for the Global Burden of Disease Study 2016. Lancet. 2017; 390: 1084-150.

4. Pandian JD, William AG, Kate MP, et al. Strategies to Improve Stroke Care Services in Low- and Middle-Income Countries: A Systematic Review. Neuroepidemiology. 2017; 49: 45-61.

5. Koennecke HC, Belz W, Berfelde D, et al. Factors influencing in-hospital mortality and morbidity in patients treated on a stroke unit. Neurology. 2011; 77: 965-72.

6. Westendorp WF, Nederkoorn PJ, Vermeij JD, Dijkgraaf MG and van de Beek D. Post-stroke infection: a systematic review and meta-analysis. BMC Neurol. 2011; 11: 110. 7. McEachern R and Campbell GD, Jr. Hospital-acquired pneumonia: epidemiology, etiology, and treatment. Infect Dis Clin North Am. 1998; 12: 761-79, x.

8. Yeh SJ, Huang KY, Wang TG, et al. Dysphagia screening decreases pneumonia in acute stroke patients admitted to the stroke intensive care unit. Journal of the Neurological Sciences. 2011; 306: 38-41.

9. Saposnik G, Hill MD, O'Donnell M, Fang J, Hachinski V and Kapral MK. Variables associated with 7-day, 30-day, and 1-year fatality after ischemic stroke. Stroke. 2008; 39: 2318-24. 
10. Finlayson O, Kapral MK, Hall R, Asllani E, Selchen D and Saposnik G. Strokeassociated pneumonia (SAP): Risk factors for its development and impact on stroke outcome. Cerebrovascular Diseases. 2010; 29: 190.

11. Finlayson O, Kapral M, Hall R, et al. Risk factors, inpatient care, and outcomes of pneumonia after ischemic stroke. Neurology. 2011; 77: 1338-45.

12. Powers WJ, Rabinstein AA, Ackerson T, et al. 2018 Guidelines for the Early Management of Patients With Acute Ischemic Stroke: A Guideline for Healthcare Professionals From the American Heart Association/American Stroke Association. Stroke. 2018.

13. Furie KL and Jayaraman MV. 2018 Guidelines for the Early Management of Patients With Acute Ischemic Stroke. Stroke. 2018.

14. Liberati A, Altman DG, Tetzlaff J, et al. The PRISMA statement for reporting systematic reviews and meta-analyses of studies that evaluate health care interventions: explanation and elaboration. Ann Intern Med. 2009; 151: W65-94.

15. Higgins JPT and Green S. Cochrane Handbook for Systematic Reviews of Interventions Version 5.1.0 [updated March 2011]. The Cochrane Collaboration, 2011. Available from www.handbook.cochrane.org. .

16. Stroup DF, Berlin JA, Morton SC, et al. Meta-analysis of observational studies in epidemiology: a proposal for reporting. Meta-analysis Of Observational Studies in Epidemiology (MOOSE) group. Jama. 2000; 283: 2008-12.

17. Ji R, Wang D, Shen H, et al. Interrelationship among common medical complications after acute stroke: Pneumonia plays an important role. Stroke. 2013; 44: 3436-44.

18. Stang A. Critical evaluation of the Newcastle-Ottawa scale for the assessment of the quality of nonrandomized studies in meta-analyses. Eur J Epidemiol. 2010; 25: 603-5. 
19. DerSimonian R and Kacker R. Random-effects model for meta-analysis of clinical trials: an update. Contemp Clin Trials. 2007; 28: 105-14.

20. Higgins JP, Thompson SG, Deeks JJ and Altman DG. Measuring inconsistency in meta-analyses. BMJ. 2003; 327: 557-60.

21. Begg CB and Mazumdar M. Operating characteristics of a rank correlation test for publication bias. Biometrics. 1994; 50: 1088-101.

22. Dziewas R, Ritter M, Schilling M, et al. Pneumonia in acute stroke patients fed by nasogastric tube. J Neurol Neurosurg Psychiatry. 2004; 75: 852-6.

23. Arnold M, Liesirova K, Broeg-Morvay A, et al. Dysphagia in Acute Stroke:

Incidence, Burden and Impact on Clinical Outcome. PLoS ONE [Electronic Resource]. 2016; 11: e0148424.

24. Lindner-Pfleghar B, Neugebauer H, Stosser S, et al. Management of dysphagia in acute stroke. A prospective study for validation of current recommendations. Der Nervenarzt. 2017; 88: 173-9.

25. Weimar C, Roth MP, Zillessen G, et al. Complications following acute ischemic stroke. European Neurology. 2002; 48: 133-40.

26. Huang WY, Weng WC, Peng TI, et al. Association of hyponatremia in acute stroke stage with three-year mortality in patients with first-ever ischemic stroke. Cerebrovasc Dis. 2012; 34: 55-62.

27. Harms H, Grittner U, Droge H and Meisel A. Predicting post-stroke pneumonia: the PANTHERIS score. Acta Neurol Scand. 2013; 128: 178-84.

28. Indredavik B, Rohweder G, Naalsund E and Lydersen S. Medical complications in a comprehensive stroke unit and an early supported discharge service. Stroke. 2008; 39: 41420. 
29. Hilker R, Poetter C, Findeisen N, et al. Nosocomial pneumonia after acute stroke: implications for neurological intensive care medicine. Stroke. 2003; 34: 975-81.

30. Hamidon BB and Raymond AA. Risk Factors and Complications of Acute Ischaemic Stroke Patients at Hospital Universiti Kebangsaan Malaysia (HUKM). Medical Journal of Malaysia. 2003; 58: 499-505.

31. Hinduja A, Dibu J, Achi E, Patel A, Samant R and Yaghi S. Nosocomial infections in patients with spontaneous intracerebral hemorrhage. Am J Crit Care. 2015; 24: 227-31.

32. Lakshminarayan K, Berger AK, Fuller CC, et al. Trends in 10-year survival of patients with stroke hospitalized between 1980 and 2000: the Minnesota stroke survey. Stroke. 2014; 45: 2575-81.

33. Chisolm D, Abegunde D and Mendis S. Scaling up action against noncommunicable diseases;How much will it cost. 2011.

34. Resar R, Pronovost P, Haraden C, Simmonds T, Rainey T and Nolan T. Using a bundle approach to improve ventilator care processes and reduce ventilator-associated pneumonia. Jt Comm J Qual Patient Saf. 2005; 31: 243-8.

35. Hellyer TP, Ewan V, Wilson P and Simpson AJ. The Intensive Care Society recommended bundle of interventions for the prevention of ventilator-associated pneumonia. J Intensive Care Soc. 2016; 17: 238-43.

36. Wip C and Napolitano L. Bundles to prevent ventilator-associated pneumonia: how valuable are they? Curr Opin Infect Dis. 2009; 22: 159-66.

37. How-to Guide: Prevent Ventilator-Associated Pneumonia; http://www.ihi.org/resources/Pages/Tools/HowtoGuidePreventVAP.aspx.

38. Grau A and Buggle F. Infection, atherosclerosis and ischemic stroke. [Spanish]. Revista de Neurologia. 1999; 29: 847-51. 
39. Kammersgaard LP, Jorgensen HS, Reith J, et al. Early infection and prognosis after acute stroke: the Copenhagen Stroke Study. J Stroke Cerebrovasc Dis. 2001; 10: 217-21. 40. Spratt N, Wang Y, Levi C, Ng K, Evans M and Fisher J. A prospective study of predictors of prolonged hospital stay and disability after stroke. J Clin Neurosci. 2003; 10: $665-9$.

41. Vargas M, Horcajada JP, Obach V, et al. Clinical consequences of infection in patients with acute stroke: is it prime time for further antibiotic trials? Stroke. 2006; 37: 4615.

42. Ros L, Garcia M, Prat J, et al. [Predictors of nosocomial infection in acute stroke. Relation with morbimortality and outcome]. Medicina Clinica. 2007; 128: 441-7.

43. Vermeij FH, Scholte op Reimer WJ, de Man P, et al. Stroke-associated infection is an independent risk factor for poor outcome after acute ischemic stroke: data from the Netherlands Stroke Survey. Cerebrovascular Diseases. 2009; 27: 465-71.

44. Minnerup J, Wersching H, Brokinkel B, et al. The impact of lesion location and lesion size on poststroke infection frequency. J Neurol Neurosurg Psychiatry. 2010; 81: 198-202. 45. Fromm A, Waje-Andreassen U, Thomassen L and Naess H. Comparison between Ischemic Stroke Patients <50 Years and >/=50 Years Admitted to a Single Centre: The Bergen Stroke Study. Stroke Res Treat. 2011; 2011: 183256.

46. Kishore AK, Vail A, Chamorro A, et al. How is pneumonia diagnosed in clinical stroke research? A systematic review and meta-analysis. Stroke (00392499). 2015; 46: 12029.

47. Westendorp WF, Vermeij JD, Zock E, et al. The Preventive Antibiotics in Stroke Study (PASS): a pragmatic randomised open-label masked endpoint clinical trial. Lancet. 2015; 385: 1519-26. 
48. Kalra L, Irshad S, Hodsoll J, et al. Prophylactic antibiotics after acute stroke for reducing pneumonia in patients with dysphagia (STROKE-INF): a prospective, clusterrandomised, open-label, masked endpoint, controlled clinical trial. Lancet. 2015; 386 North American Edition: 1835-44. 
Table 1: Characteristics of patients in studies included

\begin{tabular}{lc}
\hline Patient characteristics & $\mathbf{N}$ \\
& 139,432 \\
\hline Mean study size & 2,966 \\
Mean age of patients (years) (42 studies) & 68.3 \\
Number of male patients (44 studies) & $71,885(51.8)$ \\
Mean NIHSS score (11 studies) & 8.2 \\
Nasogastric tubes (9 studies) & $663(17 \%)$ \\
Lowered consciousness (14 studies) & $2,038(17.3 \%)$ \\
Lowered consciousness with nasogastric tube (1 study) & $21(21 \%)$ \\
Normal consciousness with nasogastric tube (1 study) & $79(79 \%)$ \\
Dysphagia (16 studies) & $1,785(22.7 \%)$ \\
Dysphagia without lowered consciousness (5 studies) & $367(52.5 \%)$ \\
Disorders of the urinary tract (4 studies) & $1,858(11 \%)$
\end{tabular}

NIHSS = National Institute of Health stroke scale 
Numbers are $\mathrm{N}(\%)$ unless otherwise indicated 
Table 2. Subgroup analyses of the pooled frequency of post-stroke pneumonia

\begin{tabular}{|c|c|c|c|c|c|c|}
\hline $\begin{array}{c}\text { Subgroup } \\
\text { classification }\end{array}$ & Subgroup & $\begin{array}{c}\text { Number of studies } \\
\text { or sample } \\
\text { populations }\end{array}$ & $\begin{array}{l}\text { Number of } \\
\text { Participants }\end{array}$ & $\begin{array}{l}\text { Pooled frequency in } \\
\text { percent }(95 \% \mathrm{CI})\end{array}$ & $\mathbf{I}^{2}$ & $\begin{array}{c}\mathbf{P} \\
\text { interaction }\end{array}$ \\
\hline \multirow[t]{5}{*}{ Patient location } & & & & & & 0.001 \\
\hline & Stroke unit & 14 & 26,942 & $8 \%(7.1 \%-9 \%)$ & $78 \%$ & \\
\hline & Ward and stroke unit & 28 & 111,409 & $13.1 \%(11.3 \%-14.9 \%)$ & $98 \%$ & \\
\hline & ICU and ward & 4 & 798 & $30.3 \%(12.9 \%-47.6 \%)$ & $97 \%$ & \\
\hline & Emergency & 1 & 283 & $9.1 \%(5.8 \%-12.5 \%)$ & - & \\
\hline \multirow[t]{4}{*}{ Type of stroke } & & & & & & 0.37 \\
\hline & Intracerebral haemorrhage & 2 & 5,423 & $16.8 \%(15.8 \%-17.8 \%)$ & $0 \%$ & \\
\hline & Ischaemic stroke & 24 & 35,252 & $11.7 \%(9.2 \%-14.1 \%)$ & $98 \%$ & \\
\hline & $\begin{array}{l}\text { Intracerebral haemorrhage } \\
\text { and ischaemic stroke }\end{array}$ & 22 & 98,757 & $11.7 \%(10.3 \%-13 \%)$ & $96 \%$ & \\
\hline \multirow[t]{4}{*}{ Era/ decade } & & & & & & 0.31 \\
\hline & $1991-2000$ & 5 & 734 & $9.9 \%(5.4 \%-14.3 \%)$ & $78 \%$ & \\
\hline & 2001- 2010 & 25 & 32,188 & $11.8 \%(9.9 \%-13.7 \%)$ & $97 \%$ & \\
\hline & 2011- 2017 & 17 & 106,406 & $13.6 \%(11.8 \%-15.3 \%)$ & $98 \%$ & \\
\hline \multicolumn{3}{|c|}{ Country/region income } & & & & 0.77 \\
\hline
\end{tabular}




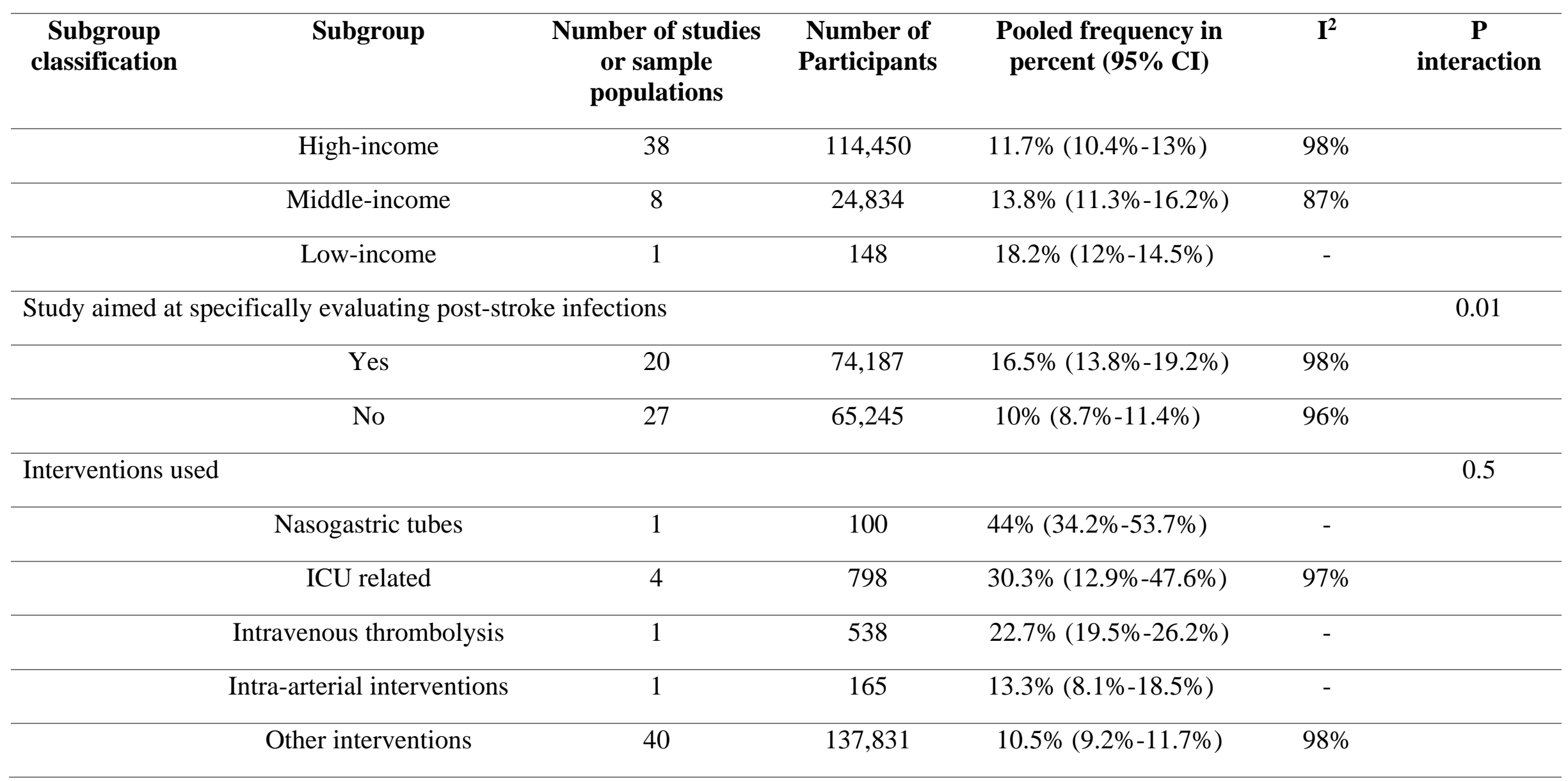


Legends for figures

Figure 1. PRISMA Flow Diagram Showing Selection of Studies

Figure 2. Quality assessment by the Newcastle-Ottawa Scale

Figure 3. Forest plot showing the pooled frequency of post-stroke pneumonia

Figure 4. Forest plot showing the pooled frequency of post-stroke infections

Figure 5. Forest plot showing the pooled frequency of post-stroke urinary tract infection 